\title{
Permeability Enhancement by Multilayer Ferromagnetic Composites for Magnetic-Core On-Chip Inductors
}

\author{
Umer Shah, Student Member, IEEE, Jessica Liljeholm, Thorbjörn Ebefors, Senior Member, IEEE, \\ and Joachim Oberhammer, Senior Member, IEEE
}

\begin{abstract}
This paper reports about un-patterned ferromagnetic NiFe/AIN multilayer composites used as advanced magnetic core materials for on-chip and interposer integrated inductances. The proposed composite structure reduces RF induced currents and thus pushes the permeability cut-off to beyond $3.7 \mathrm{GHz}$, which is by a factor of 7.1 higher than for homogeneous NiFe layers of same thickness. To the best knowledge of the authors, we achieve the highest effective relative permeability of 28 at $1 \mathrm{GHz}$, highest ferromagnetic resonance frequency and highest inductance enhancement factor above $1 \mathrm{GHz}$ ever reported for devices based on on-chip un-patterned $\mathrm{NiFe}$ magnetic cores. A single loop inductor is also implemented as a technology demonstrator, achieving an inductance enhancement of 4.8 and a quality factor enhancement of 4.5 at $400 \mathrm{MHz}$.
\end{abstract}

Index Terms-Magnetic materials, microstrip, NiFe multilayer composite, permeability, permittivity, micromachined inductors.

\section{INTRODUCTION}

$\mathbf{O}$ $\mathrm{N}$-CHIP and interposer integrated inductors, used as passive components in RFICs or in system-in-package integrated components, are currently employed as air-core inductors with a maximum inductance density of $200 \mathrm{nH} / \mathrm{mm}^{2}$ [1]. Their inductance per area would significantly be enhanced by magnetic core materials. At lower frequencies (DC to $10 \mathrm{MHz}$ ), the relative permeability of sputtered $\mathrm{NiFe}$ layers can be as high as 3000 , and with an optimized electrodeposition process even a permeability of 8500 was recently achieved [2]. However, such homogeneous NiFe films suffer from a number of limitations at frequencies above $100 \mathrm{MHz}$. Firstly, the magnetic NiFe layers are metallic and thus conductive which results in induced currents counteracting the magnetic field, increasing losses and reducing the effective inductance. Secondly, they have low ferromagnetic resonance frequency. To suppress the flow of eddy currents in these films only very thin NiFe layers are used. For inductor cores, larger magnetic volumes and thus thicker layers are desired. Placing

The authors acknowledge the process development support by Oerlikon Systems, in particular Harmut Rohrmann, and by Vincent Ip and Katarina Rook of Veeco Instruments, and the financial support by VINNOVA under grant no. 2012-00515.

Umer Shah and Joachim Oberhammer are with the Department of Micro and Nanosystems, KTH Royal Institute of Technology, Stockholm SE-100 44, Sweden (e-mail: umers@kth.se; joachim.oberhammer@ee.kth.se).

Jessica Liljeholm and Thorbjörn Ebefors are with SILEX Microsystems AB, Järfälla, Sweden (e-mail: jessica.liljeholm@silex.se; thorbjorn.ebefors@silex.se).

Manuscript received March 26, 2014; revised June 24, 2014

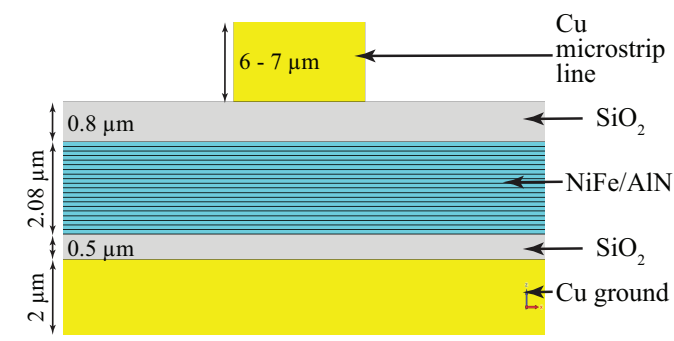

Fig. 1. Schematic cross-section of the microstrip test structure fabricated at $200 \mathrm{~mm}$ MEMS foundry.

the NiFe film between isolation layers suppresses the induced currents in the $\mathrm{NiFe}$ film and at the same time increases the ferromagnetic resonance [3], [4]. Patterning the magnetic film enhances the anisotropy which increases the ferromagnetic resonance [5]. Another approach to increase the resistivity and ferromagnetic resonance is to use granular films which add to the complexity of processing. The eddy currents can also be reduced by using a magnetic nanoparticle composite, but only a rather low permeability and an inductance enhancement of less than 1.5 was reported [6].

$\mathrm{NiFe} / \mathrm{SiO}_{2}$ multilayer films in [7] achieved a ferromagnetic resonance frequency above $5 \mathrm{GHz}$ with an inductance improvement of only $40 \%$, but for achieving this performance, patterning of the layers was required. A quality factor improvement of $30 \%$ could be achieved when applying a DC magnetic bias field during measurements which is undesirable in integrated solutions. $\mathrm{NiFe} / \mathrm{Cr}$ multilayer films have been used in [8], [9] to enhance the inductance by a factor of 6 and the quality factor by a factor of 3 at $200 \mathrm{MHz}$. However, the ferromagnetic resonance frequency achieved was just above $1 \mathrm{GHz}$ since chromium, being a conductive material, does not suppress induced currents in the $\mathrm{NiFe}$ layers. $\mathrm{IrMn} / \mathrm{CoFe}$ antiferromagnetic/ferromagnetic multilayers stack used in [10] achieved a ferromagnetic resonance frequency of $4.3 \mathrm{GHz}$ but the inductance and the quality factor enhancement were only $20 \%$ and $6.8 \%$, respectively.

In the present paper, an un-patterned ferromagnetic $\mathrm{NiFe} / \mathrm{AlN}$ sandwich structure is used as magnetic core material where the AlN interlayers suppress the induced currents very effectively, provides stress compensation for $\mathrm{NiFe}$ layers and at the same time increases the ferromagnetic resonance to beyond 3.7 GHz. This results in the highest effective relative permeability and highest inductance enhancement factor achieved 


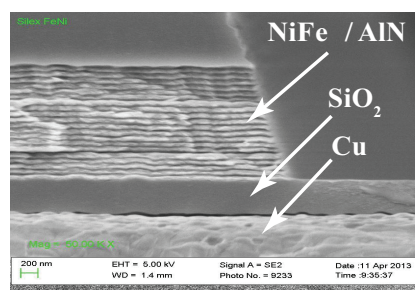

(a)

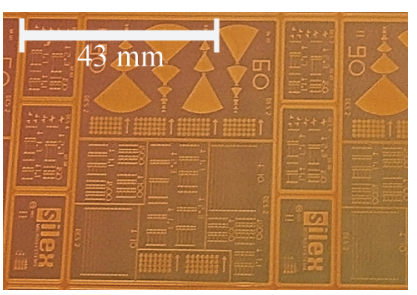

(b)

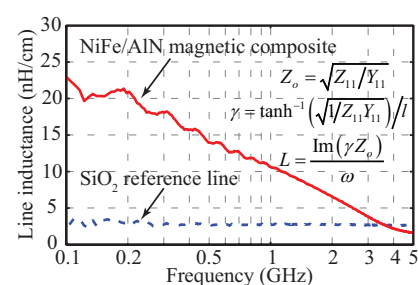

(a)

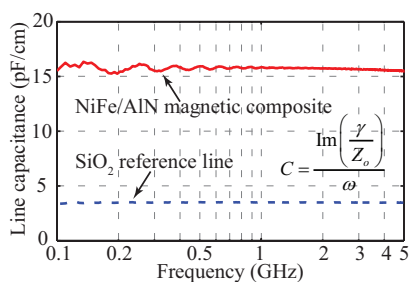

(b)
Fig. 2. Magnetic NiFe/AlN multilayer composite structures: (a) SEM image; and (b) photograph of part of the wafer showing the fabricated test structures.

above $1 \mathrm{GHz}$ for un-patterened NiFe layers.

\section{CONCEPT, DESIGN AND FABRICATION}

A micromachined microstrip configuration was chosen as the test structure (top copper microstrip line, the composite ferromagnetic material core, a bottom copper ground layer). The ferromagnetic material chosen for this evaluation is nickeliron (Ni-Fe) because of its common use in semiconductor fabrication, in particular for magnetic sensors and hard disk read-write heads. Between the individual NiFe layers, thin aluminum nitride (AlN) insulation layers are inserted to suppress leakage and induced currents flowing in the ferromagnetic core layer which results in an increase in the ferromagnetic resonance. In addition, the AlN layers act as stress compensation for the sputtered NiFe layers, much more efficiently than $\mathrm{SiO}_{2}$ insulating films. The fabrication was done in a commercial $200 \mathrm{~mm}$ wafer foundry. The magnetic film is deposited in an Oerlikon LLS sputter tool, and consists of $100 \mathrm{~nm}$ thick $\mathrm{NiFe}$ layers intersected by $8 \mathrm{~nm}$ thick AlN insulation layers, embedded between a top and a bottom PECVD $\mathrm{SiO}_{2}$ layer as shown in Fig. 1. The total thickness of the NiFe/AlN multilayer composite is $2.08 \mu \mathrm{m}$ and was chosen as a compromise between the total deposition time of the layered stack and significant RF performance enhancement. The thickness of $100 \mathrm{~nm}$ chosen for individual NiFe layers is a good compromise for achieving high total volume ratio of $\mathrm{NiFe}$, by still limiting the induced RF currents within the individual layers [1]. Vertical interconnects were created by etching the magnetic/dielectric layers in a Veeco Nexus IBE dry ion beam etching tool. The NiFe stack was insitu magnetized during sputter deposition. Fig. 2 shows a SEM image of the cross-section and a photograph of the fabricated test structures. From the measured S-parameters of the microstrip lines, the frequency-dependent characteristic impedance and propagation constant are extracted. The inductance and capacitance per unit length are subsequently derived by using the formulas shown in Fig. 3(a) and Fig. 3(b).

\section{EXPERIMENTAL RESULTS}

The inductance per unit length of the microstrip line with NiFe/AlN multilayer composite is plotted in Fig. 3(a) together with a $\mathrm{SiO}_{2}$ reference line of equivalent thickness. The higher inductance is directly related to the high effective relative permeability of the magnetic stack. The calculated capacitance per unit length is plotted in Fig. 3(b) together with the $\mathrm{SiO}_{2}$
Fig. 3. Measurement of microstrip lines with and without the ferromagnetic $\mathrm{NiFe} / \mathrm{AlN}$ multilayer composite: (a) inductance per unit length vs frequency ; and (a) capacitance per unit length vs frequency.

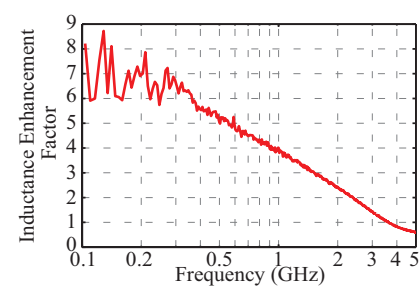

(a)

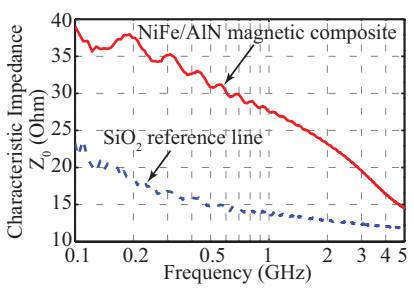

(b)
Fig. 4. Measurement of the microstrip line with ferromagnetic NiFe/AlN multilayer composite relative to an $\mathrm{SiO}_{2}$ reference line: (a) inductance enhancement factor vs frequency; and (b) characteristic impedance.

reference line. The larger capacitance per unit length compared to the reference line is due to the NiFe/AlN multilayer composite whose multiple capacitances result in much wider fringing field volume and since the effective total thickness of the capacitance is reduced by the many conducting layers. The ferromagnetic resonance frequency is not affected by the larger line capacitance as it is independent of the line capacitance. The implications of a larger line capacitance on the self resonance frequency of any designed inductor is difficult to predict as the increase in line capacitance is accompanied by an increase in inductance which allows for a smaller geometry with reduced capacitance.

Fig. 4(a) shows the inductance enhancement factor for the NiFe/AlN multilayer composite line, which is about 4 at $1 \mathrm{GHz}$ relative to a $\mathrm{SiO}_{2}$ reference line. The inductance enhancement factor for a conductor is proportional to the portion of its magnetic flux path that is filled with magnetic material [7]. In our microstrip configuration, the magnetic field lines below the signal line are completely confined within the composite magnetic material which results in a higher inductance enhancement factor, as compared to having the magnetic stack on top of a planar conductor as implemented in [7], [10], with lower inductance enhancement factor due to a smaller portion of the magnetic flux being confined in the magnetic volume. Fig. 4(b) shows the characteristic impedance of the microstrip line with the $\mathrm{NiFe} / \mathrm{AlN}$ multilayer composite together with the $\mathrm{SiO}_{2}$ reference line. The frequency dependent effective relative permeability of the NiFe/AlN multilayer composite material is shown in Fig. 5. It was extracted from the measurements with help of an Ansoft HFSS simulation model, by parameter matching of the material permeability in the model until the the same inductance per unit length as measured is achieved, done individually for all frequency 


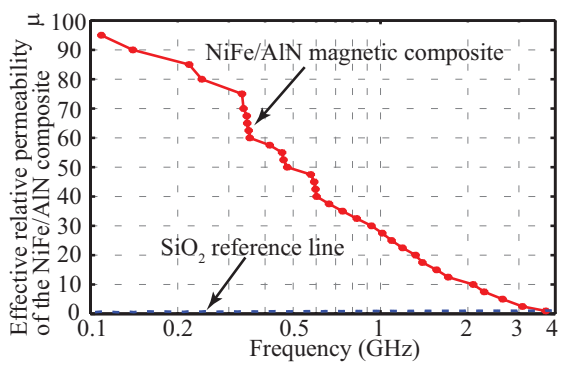

Fig. 5. Effective relative permeability of the ferromagnetic NiFe/AlN multilayer composite relative to $\mathrm{SiO}_{2}$ extracted from the measured S-parameters.

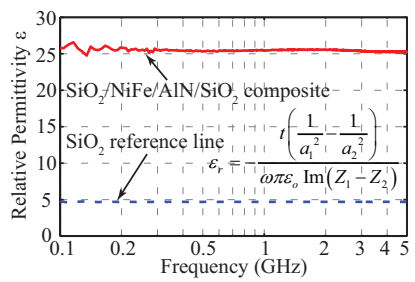

(a)

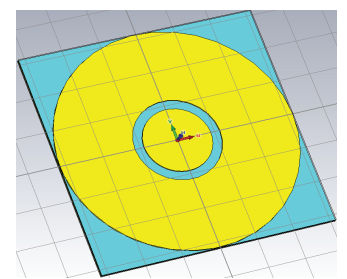

(b)
Fig. 6. Permittivity of the ferromagnetic NiFe/AlN multilayer composite relative to $\mathrm{SiO}_{2}$ line extracted from the measured S-parameters: (a) relative permittivity; and (b) capacitive disc used to extract permittivity.

points, as the permeability decreases with frequency. An effective relative permeability of 28 was achieved at $1 \mathrm{GHz}$, and the ferromagnetic resonance exceeds $3.7 \mathrm{GHz}$. The extracted relative permeability of the reference line is also shown with a nearly-constant value of 1 throughout the frequency range of interest, confirming the permeability extraction method.

The measured frequency-dependent relative permittivity of the $\mathrm{SiO}_{2} / \mathrm{NiFe} / \mathrm{AlN} / \mathrm{SiO}_{2}$ multilayer composite material is shown in Fig. 6(a) together with the measured relative permittivity of the $\mathrm{SiO}_{2}$ insulation layer of the reference line. The extracted permittivity of the reference line agrees very well with reported permittivities of $\mathrm{SiO}_{2}$. The capacitive structure used to measure the permittivity is shown in an insert in the figure. Disc radii of $75 \mu \mathrm{m}$ and $90 \mu \mathrm{m}$ are used for the test structures in this frequency range [11].

\section{TEST INDUCTOR}

A single-loop microstrip test inductor was implemented by a $2.3 \mathrm{~mm}$ long and $5 \mu \mathrm{m}$ wide microstrip line which is shortcircuited at one end to its ground layer. The resulting very shallow inductor loop, as shown in Fig. 7(a), has a poor area to loop-length ratio and therefore low Q. Still, this test inductor demonstrated an inductance enhancing factor of 4.8 and a quality factor enhancement factor of 4.5 measured at $400 \mathrm{MHz}$ of the magnetic stack as compared to a reference loop using a $\mathrm{SiO}_{2}$ dielectric layer. The vertical transition between the metal layers was determined by four-probe measurements to be $4.1 \mathrm{~m} \Omega$.

\section{CONCLUSION}

In conclusion, the proposed un-patterned multilayer $\mathrm{NiFe} / \mathrm{AlN}$ composite material enables an increase of the ferromagnetic resonance and thus an enhancement in the

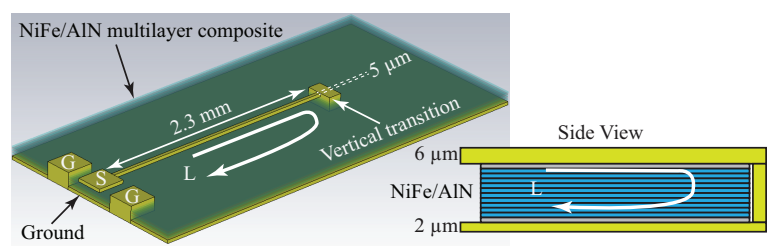

(a)

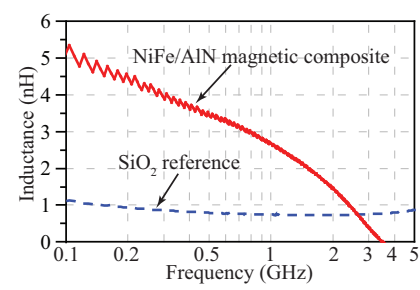

(b)

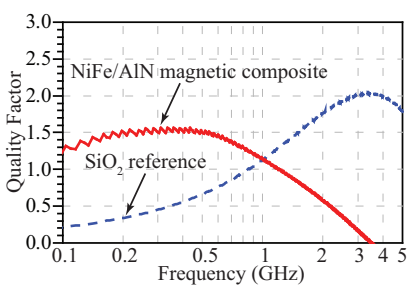

(c)
Fig. 7. Inductor measurements with and without the ferromagnetic NiFe/AlN multilayer composite: (a) inductor layout; (b) extracted inductance; and (c) extracted quality factor.

frequency utilization by a factor of 7.1 as compared to previous work of homogeneous NiFe films of similar total thicknesses, and the so far highest permeability values above $1 \mathrm{GHz}$ for on-chip integrated un-patterned NiFe layers were achieved. Compared to [7], we achieve a higher inductance and quality factor enhancement. Compared to [8], [9], we achieve a higher quality factor enhancement, a higher ferromagnetic resonance frequency and a higher inductance enhancement above $500 \mathrm{MHz}$.

\section{REFERENCES}

[1] D. S. Gardner, G. Schrom, F. Paillet, B. Jamieson, T. Karnik, and S. Borkar, "Review of on-chip inductor structures with magnetic films," IEEE Trans. Magn., vol. 45, no. 10, pp. 4760-4766, Oct. 2009.

[2] M. Glickman, T. Niblock, J. Harrison, I. B. Goldberg, P. Tseng, and J. W. Judy, "High permeability permalloy for MEMS," in Proc. Hilton Head, 2010.

[3] Y. Zhuang, M. Vroubel, B. Rejaei, E. Boellaard, and J. N. Burghartz, "Investigation of microstrips with $\mathrm{NiFe}$ magnetic thin film (I): Experiment," Trans. Magn. Soc. Jpn., vol. 2, no. 5, pp. 367-370, 2002.

[4] M. Vroubel, Y. Zhuang, B. Rejaei, and J. N. Burghartz, "Investigation of microstrips with NiFe magnetic thin film (II): Modeling," Trans. Magn. Soc. Jpn., vol. 2, no. 5, pp. 371-376, 2002.

[5] P. K. Amiri, "Magnetic materials and devices for integrated radio-frequency electronics," Ph.D. dissertation, Delft University of Technology, Oct. 2008. [Online]. Available: http://repository.tudelft.nl/ view/ir/uuid:2d9d87dd-cb7c-4919-8fbb-595d9a8353f1/

[6] K. Koh, J. Park, J. Park, X. Zhu, and L. Lin, "Core-shell magnetic nanoparticles for on-chip RF inductors," in IEEE Int. Conf. Micro Electro Mechanical Systems, Jan. 2013, pp. 465-468.

[7] J. Salvia, J. A. Bain, and C. P. Yue, "Tunable on-chip inductors up to $5 \mathrm{GHz}$ using patterned permalloy laminations," in IEEE Int. Electron Devices Meeting, Dec. 2005, pp. 943-946.

[8] T. Dastagir, W. Xu, S. Sinha, H. Wu, Y. Cao, and H. Yu, "Tuning the permeability of permalloy films for on-chip inductor applications," Appl. Phys. Lett., vol. 97, no. 16, pp. 162 506-162 506-3, Oct. 2010.

[9] W. Xu, S. Sinha, T. Dastagir, H. Wu, B. Bakkaloglu, D. S. Gardner, Y. Cao, and H. Yu, "Performance enhancement of on-chip inductors with permalloy magnetic rings,' IEEE Electron Device Lett., vol. 32, no. 1, pp. 69-71, Jan. 2011.

[10] R.-F. Jiang, N. N. Shams, M. T. Rahman, and C.-H. Lai, "Exchangecoupled irmn/cofe mulitlayers for RF-integrated inductors," IEEE Trans. Magn., vol. 43, no. 10, pp. 3930-3932, Oct. 2007.

[11] Z. Ma, A. J. Becker, P. Polakos, H. Huggins, J. Pastalan, H. Wu, K. Watts, Y. H. Wong, and P. Mankiewich, "RF measurement technique for characterizing thin dielectric films," IEEE Trans. Electron Devices, vol. 45, no. 8, pp. 1811-1816, Aug. 1998. 